\title{
The Generalized Weierstrass System for Nonconstant Mean Curvature Surfaces and the Nonlinear Sigma Model
}

\author{
Paul Bracken \\ Department of Mathematics, \\ University of Texas, \\ Edinburg, TX
}

$78541-2999$

\begin{abstract}
A study of the generalized Weierstrass system which can be used to induce mean curvature surfaces in three-dimensional Euclidean space is presented. A specific transformation is obtained which reduces the initial system to a two-dimensional Euclidean nonlinear sigma model. Some aspects of integrability are discussed, in particular, a connection with a version of the sinh-Gordon equation is established. Finally, some specific solutions are given and a systematic way of calculating multisoliton solutions is presented.
\end{abstract}




\section{Introduction.}

The method which was first formulated by Weierstrass and Enneper [1] for determining minimal surfaces embedded in three-dimensional Euclidean space has recently been generalized by B. Konopelchenko $[2,3]$ and it has been the subject of considerable further work [4-7]. One of the reasons for this is that there are many physical applications of minimal surfaces to such areas as integrable systems, statistical mechanics and even string theory [5,8]. In fact, a direct connection between certain classes of mean curvature surface, namely constant mean curvature, and a particular integrable finite dimensional Hamiltonian system has been established by Konopelchenko and Taimanov [3]. Mean curvature plays a special role among the characteristics of surfaces and their dynamics in many problems that arise in both physics and mathematics [8]. The case in which the mean curvature of the surface is constant has been discussed [4-5] at length. In particular, many properties of such surfaces determined by Konopelchenko's inducing prescription, such as the relationship to the nonlinear two-dimensional sigma model, integrability and Lax pair have been determined and a Bäcklund transformation has been calculated as well [6-7].

It is the purpose here to begin an investigation of the case in which the mean curvature of the surface is not constant, but is described by a real-valued function which depends on two independent variables.

This is useful for several reasons, in particular for establishing some of the mathematical properties of such surfaces in general, beyond the case of constant mean curvature surfaces. There are many applications of mean curvature surfaces and, of particular interest here, we elaborate on applications to the areas of quantum field theory, two-dimensional gravity and string theory $[9-10]$. Classical string theory can be regarded as a study of the geometry of subspaces immersed in higher dimensional spaces of manifolds. The variational equations of motion for the string or membrane involve the mean curvature function. These equations are usually obtained by varying a particular functional, such as the Willmore functional, which depends explicitly on the mean curvature. Once this is determined, the present approach would provide a way of associating a particular surface with the particular mean curvature function. The three-dimensional case has been discussed somewhat and may not be the most significant in physical applications, but it is 
useful to study and will hopefully lead the way to generalizations to higher dimensional spaces. Thus, a more sophisticated approach would involve the calculation of the mean curvature from the proposed equations of motion, which result from the variation of some given action functional. This results in a function that can be employed in an inducing procedure such as the one described here to generate a surface in a higher dimensional space. Generating the surface then amounts to solving a system of first-order nonlinear partial differential equations which depend on the mean curvature function for a set of functions. These functions can be used to determine the coordinates of a surface in Euclidean three space corresponding to the given mean curvature function by means of a particular inducing prescription. One in particular which is relevant to the case of three space will be presented here. A connection between the generalized Weierstrass system and a modified sigma model system is established. This connection allows solutions of the sigma model system to be related to solutions of the generalized Weierstrass system which can be used to produce surfaces. A set of conservation laws relevant to the generalized Weierstrass system is presented. Integrability is reviewed and a connection with the sinh-Gordon equation is made. Finally, some specific solutions are determined for the sigma model system for specific mean curvature and the corresponding solutions to the generalized Weierstrass system are determined from these as well.

\section{First Order System and Inducing Formulas}

The set of functions which are responsible for determining the surface corresponding to a mean curvature function $H$ will be determined from a system of coupled first order differential equations. The following nonlinear Dirac-type system of differential equations which determine two complex-valued functions $\psi_{1}$ and $\psi_{2}$ corresponding to the mean curvature function $H$ are used to generate a surface immersed in $\mathbb{R}^{3}$. This system is given by Konopelchenko [2-3] in complex form as follows

$$
\begin{gathered}
\partial \psi_{1}=p H \psi_{2}, \quad \bar{\partial} \psi_{2}=-p H \psi_{1}, \\
\bar{\partial} \bar{\psi}_{1}=p H \bar{\psi}_{2}, \quad \partial \bar{\psi}_{2}=-p H \bar{\psi}_{1}, \\
p=\left|\psi_{1}\right|^{2}+\left|\psi_{2}\right|^{2},
\end{gathered}
$$

where $\psi_{1}$ and $\psi_{2}$ are two complex functions of the complex variables $(z, \bar{z})$. The bar denotes complex conjugation in what follows and we define the derivatives $\partial=\partial / \partial z$ and $\bar{\partial}=\partial / \partial \bar{z}$. 
The function $H=H(z, \bar{z})$ denotes the mean curvature of the surface. The system (2.1) can be considered as a variant of the original Weierstrass-Enneper system [1]. Moreover, system (2.1) determines a set of constant mean curvature surfaces which are obtained by means of the following parametrization $(z, \bar{z}) \rightarrow\left(X_{1}(z, \bar{z}), X_{2}(z, \bar{z}), X_{3}(z, \bar{z})\right)$, such that the $X_{j}$ can be determined explicitly from the solutions $\psi_{i}$ to $(2.1)$ by evaluating the following set of integrals

$$
\begin{aligned}
& X_{1}+i X_{2}=2 i \int_{z_{0}}^{z}\left(\bar{\psi}_{1}^{2} d z^{\prime}-\psi_{2}^{2} d \bar{z}^{\prime}\right) \\
& X_{1}-i X_{2}=2 i \int_{z_{0}}^{z}\left(\psi_{2}^{2} d z^{\prime}-\psi_{1}^{2} d \bar{z}^{\prime}\right) \\
& X_{3}=-2 \int_{z_{0}}^{z}\left(\bar{\psi}_{1} \psi_{2} d z^{\prime}+\psi_{1} \bar{\psi}_{2} d \bar{z}^{\prime}\right) .
\end{aligned}
$$

The Gaussian curvature of the surface is then given by

$$
K=-\frac{\partial \bar{\partial} \log p}{p^{2}}
$$

where $p$ is given in (2.1) in terms of the $\psi_{i}$. In $(2.1)$, the function $H(z, \bar{z})$ is arbitrary, and so, for given $H(z, \bar{z})$, a particular solution to (2.1) will generate a surface in Euclidean three space by means of equations (2.2).

\section{Associated Second Order System.}

It is useful to subject the system described in (2.1) to several transformations in order to investigate its structure. In fact, it will be shown that (2.1) has an associated second order system whose solutions can be used to determine surfaces by means of a transformation linking these solutions to corresponding solutions of (2.1). Begin by introducing the new complex variable

$$
\rho=\frac{\psi_{1}}{\bar{\psi}_{2}}
$$

where $\psi_{1}$ and $\psi_{2}$ satisfy system (2.1). To calculate $\partial \rho$, we differentiate (3.1) and, upon using the relation $p=\left|\psi_{2}\right|^{2}\left(1+|\rho|^{2}\right)$, we obtain

$$
\partial \rho=\frac{\partial \psi_{1}}{\bar{\psi}_{2}}-\frac{\psi_{1}}{\bar{\psi}_{2}^{2}} \partial \bar{\psi}_{2}=\frac{p H}{\bar{\psi}_{2}^{2}}\left(\left|\psi_{1}\right|^{2}+\left|\psi_{2}\right|^{2}\right)=\frac{H p^{2}}{\bar{\psi}_{2}^{2}}=H \psi_{2}^{2}\left(1+|\rho|^{2}\right)^{2} .
$$

Solving this equation for $\psi_{2}$, we find that

$$
\psi_{2}=\epsilon \frac{(\partial \rho)^{1 / 2}}{H^{1 / 2}\left(1+|\rho|^{2}\right)}, \quad \epsilon= \pm 1
$$


From (3.1), it is easy to determine $\psi_{1}$ to be

$$
\psi_{1}=\epsilon \frac{\rho(\bar{\partial} \bar{\rho})^{1 / 2}}{H^{1 / 2}\left(1+|\rho|^{2}\right)}, \quad \epsilon= \pm 1
$$

This generates the following transformation from the variable $\rho$ into the pair of variables $\psi_{i}$ provided that the $\psi_{i}$ satisfy (2.1). To summarize, we have shown that

$$
\psi_{1}=\epsilon \rho \frac{(\bar{\partial} \bar{\rho})^{1 / 2}}{H^{1 / 2}\left(1+|\rho|^{2}\right)}, \quad \psi_{2}=\epsilon \frac{(\partial \rho)^{1 / 2}}{H^{1 / 2}\left(1+|\rho|^{2}\right)}, \quad \epsilon= \pm 1 .
$$

Proposition 1. If $\psi_{1}$ and $\psi_{2}$ are solutions of system (1.1), then the function $\rho$ defined by (3.1) and related to $\psi_{1}$ and $\psi_{2}$ by (3.3) is a solution of the following second order system

$$
\partial \bar{\partial} \rho-\frac{2 \bar{\rho}}{1+|\rho|^{2}} \partial \rho \bar{\partial} \rho=\bar{\partial}(\ln H) \partial \rho, \quad \bar{\partial} \partial \bar{\rho}-\frac{2 \rho}{1+|\rho|^{2}} \bar{\partial} \bar{\rho} \partial \bar{\rho}=\partial(\ln H) \bar{\partial} \bar{\rho}
$$

Proof: Differentiating the equation $\partial \rho=H p^{2} / \bar{\psi}_{2}^{2}$ with respect to $\bar{z}$, we obtain that

$$
\begin{gathered}
\bar{\partial} \partial \rho=\frac{\bar{\partial} H p^{2}}{\bar{\psi}_{2}^{2}}+2 H \frac{p \bar{\partial} p}{\bar{\psi}_{2}^{2}}-2 H \frac{p^{2}}{\bar{\psi}_{2}^{3}}\left(\bar{\partial} \bar{\psi}_{2}\right) \\
=\frac{H_{p^{2}}^{2}}{\bar{\psi}_{2}^{2}}\left[\bar{\partial}(\ln H)+\frac{2}{p \bar{\psi}_{2}}\left(\bar{\psi}_{1} \bar{\psi}_{2}\left(\bar{\partial} \psi_{1}\right)+\left|\psi_{2}\right|^{2}\left(\bar{\partial} \bar{\psi}_{2}\right)-\left|\psi_{1}\right|^{2} \bar{\partial} \bar{\psi}_{1}-\left|\psi_{2}\right|^{2} \bar{\partial} \bar{\psi}_{2}\right)\right] \\
=\frac{p H}{\bar{\psi}_{2}^{3}}\left[p \bar{\psi}_{2} \bar{\partial}(\ln H)+2\left(\bar{\psi}_{1} \bar{\psi}_{2}\left(\bar{\partial} \psi_{1}\right)-\left|\psi_{1}\right|^{2}\left(\bar{\partial} \bar{\psi}_{2}\right)\right)\right] .
\end{gathered}
$$

Substituting the derivatives into the left hand side of (3.4), we have

$$
\begin{gathered}
\partial \bar{\partial} \rho-\frac{2 \bar{\rho}}{1+|\rho|^{2}} \partial \rho \bar{\partial} \rho \\
=\frac{p H}{\bar{\psi}_{2}^{3}}\left[p \overline{\psi_{2}} \bar{\partial}(\ln H)+2 \bar{\psi}_{1} \bar{\psi}_{2}\left(\bar{\partial} \psi_{1}\right)-2\left|\psi_{1}\right|^{2}\left(\bar{\partial} \bar{\psi}_{2}\right)\right]-\frac{2\left(\bar{\psi}_{1} / \psi_{2}\right)\left|\psi_{2}\right|^{2}}{\left|\psi_{1}\right|^{2}+\left|\psi_{2}\right|^{2}} \frac{p^{2}}{\bar{\psi}_{2}^{4}}\left(\bar{\psi}_{2} \bar{\partial} \psi_{1}-\psi_{1} \bar{\partial} \bar{\psi}_{2}\right) \\
=\frac{p H}{\bar{\psi}_{2}^{3}}\left[p \bar{\psi}_{2} \bar{\partial}(\ln H)+2 \bar{\psi}_{1} \bar{\psi}_{2}\left(\bar{\partial} \psi_{1}\right)-2\left|\psi_{1}\right|^{2} \bar{\partial} \bar{\psi}_{2}-2 \bar{\psi}_{1} \bar{\psi}_{2} \bar{\partial} \psi_{1}+2\left|\psi_{1}\right|^{2} \bar{\partial} \bar{\psi}_{2}\right] \\
=\frac{p^{2} H}{\bar{\psi}_{2}^{2}} \bar{\partial}(\ln H)=(\bar{\partial} \ln H) \partial \rho .
\end{gathered}
$$

The second conjugate equation in (3.4) can be obtained in the same way. QED

There is a converse to Proposition 1, and this is formulated in the following statement.

Proposition 2. If $\rho$ is a solution to system (3.4), then the functions $\psi_{1}$ and $\psi_{2}$ defined in (3.3) in terms of $\rho$ satisfy $(2.1)$. 
Proof: Differentiate $\psi_{1}$ given by (3.3) with respect to $z$ and eliminate the second derivative $\partial \bar{\partial} \bar{\rho}$ using (3.4) to obtain

$$
\begin{gathered}
\partial \psi_{1}=\epsilon \partial \rho \frac{(\bar{\partial} \bar{\rho})^{1 / 2}}{H^{1 / 2}\left(1+|\rho|^{2}\right)}+\frac{1}{2} \frac{\epsilon \rho}{H^{1 / 2}\left(1+|\rho|^{2}\right)}\left(\frac{2 \rho}{1+|\rho|^{2}} \partial \bar{\rho}+\partial(\ln H)\right)(\bar{\partial} \bar{\rho})^{1 / 2} \\
-\frac{1}{2} \epsilon \rho \frac{(\bar{\partial} \bar{\rho})^{1 / 2} \partial(\ln H)}{H^{1 / 2}\left(1+|\rho|^{2}\right)}-\epsilon \rho \frac{(\bar{\partial} \bar{\rho})^{1 / 2}}{H^{1 / 2}\left(1+|\rho|^{2}\right)^{2}}(\partial \rho \bar{\rho}+\partial \bar{\rho} \rho) \\
=\epsilon \partial \rho\left(1+|\rho|^{2}\right) \frac{(\bar{\partial} \bar{\rho})^{1 / 2}}{H^{1 / 2}\left(1+|\rho|^{2}\right)^{2}}-\epsilon|\rho|^{2} \partial \rho \frac{(\bar{\partial} \bar{\rho})^{1 / 2}}{H^{1 / 2}\left(1+|\rho|^{2}\right)^{2}}=H(z, \bar{z}) p \psi_{2} .
\end{gathered}
$$

The other equation in (2.1) is developed by differentiating $\psi_{2}$ in (3.3) with respect to $\bar{z}$ and simplifying as in the case presented above.

There are several conservation laws as well associated with system (2.1) which are important and need to be discussed. One reason is that these results will imply the path independence of the integrals which generate the coordinate functions of the surface given in (2.2). It can be noted that the system (3.4) is invariant under discrete transformations generated by the reflections

$$
\begin{aligned}
& Z_{1}: \quad z \rightarrow z, \quad \bar{z} \rightarrow-\bar{z}, \quad \rho \rightarrow \rho, \quad \bar{\rho} \rightarrow \bar{\rho}, \\
& Z_{2}: \quad z \rightarrow z, \quad \bar{z} \rightarrow \bar{z}, \quad \rho \rightarrow-\rho, \quad \bar{\rho} \rightarrow-\bar{\rho}, \\
& Z_{3}: \quad z \rightarrow-z \quad \bar{z} \rightarrow \bar{z}, \quad \rho \rightarrow-\rho, \quad \bar{\rho} \rightarrow-\bar{\rho},
\end{aligned}
$$

their complex conjugates as well as the inversion

$$
I: z \rightarrow z, \quad \bar{z} \rightarrow \bar{z} \quad \rho \rightarrow 1 / \rho, \quad \bar{\rho} \rightarrow 1 / \bar{\rho}
$$

There is the conservation of a potential function with respect to (2.1), namely,

$$
\partial\left(\psi_{1}^{2}\right)+\bar{\partial}\left(\psi_{2}^{2}\right)=2 \psi_{1} \partial \psi_{1}+2 \psi_{2} \bar{\partial} \psi_{2}=2 \psi_{1} p H \psi_{2}+2 \psi_{2}\left(-p H \psi_{1}\right)=0
$$

The conjugate of this equation holds as well. Moreover, there is a further conservation relation

$$
\partial\left(\psi_{1} \bar{\psi}_{2}\right)+\bar{\partial}\left(\bar{\psi}_{1} \psi_{2}\right)=0
$$

Next, let us define the quantity

$$
J=\bar{\psi}_{1} \partial \psi_{2}-\psi_{2} \partial \bar{\psi}_{1}
$$


Differentiating $J$ in $(3.7)$ with respect to $\bar{z}$, we find that

$$
\begin{gathered}
\bar{\partial} J=\bar{\partial} \bar{\psi}_{1} \partial \psi_{2}+\bar{\psi}_{1} \bar{\partial} \partial \psi_{2}-\bar{\partial} \psi_{2} \partial \bar{\psi}_{1}-\psi_{2} \bar{\partial} \partial \bar{\psi}_{1} \\
=p H \bar{\psi}_{2} \partial \psi_{2}+\bar{\psi}_{1} \partial\left(-p H \psi_{1}\right)+p H \psi_{1} \partial \bar{\psi}_{1}-\psi_{2} \partial\left(p H \bar{\psi}_{2}\right) \\
=p H \bar{\psi}_{2} \partial \psi_{2}-\bar{\psi}_{1}\left(\partial H p \psi_{1}+H \partial p \psi_{1}+p H \partial \psi_{1}\right)+p H \psi_{1} \partial \bar{\psi}_{1} \\
-\psi_{2}\left(\partial H p \bar{\psi}_{2}+H \partial p \bar{\psi}_{2}+p H \partial \bar{\psi}_{2}\right) \\
=p H(\partial p)-p^{2} \partial H-p H \partial p-p H \bar{\psi}_{1}\left(p H \psi_{2}\right)-p H \psi_{2}\left(-p \bar{\psi}_{1}\right)=-p^{2}(\partial H) .
\end{gathered}
$$

Clearly, if $H$ is constant then $\bar{\partial} J=0$, in which case $J$ is a conserved quantity, or current. Nonconstant $H$ breaks the conservation of this current and $J$ given by (3.7) is no longer conserved. However, using the result (3.8), we can modify $J$ to a new form which is conserved under differentiation by $\bar{z}$.

Proposition 3. The quantity defined in terms of $J$ from (3.7) in the form

$$
\mathcal{J}=J+\int_{\bar{z}_{0}}^{\bar{z}} p^{2}(z, \tau) \partial H(z, \tau) d \tau
$$

where $\bar{z}_{0}$ is fixed in $\mathbb{C}$ is conserved under differentiation with respect to $\bar{z}$.

Proof: To prove this, differentiate (3.9) with respect to $\bar{z}$ and use (3.8) to obtain

$$
\bar{\partial} \mathcal{J}=\bar{\partial} J+p^{2}(z, \bar{z}) \partial H(z, \bar{z})=-p^{2}(\partial H)+p^{2}(\partial H)=0
$$

QED

Therefore, $\mathcal{J}$ defined by (3.9) is conserved with respect to differentiation with respect to $\bar{z}$. We can represent the integral operator used in (3.9) by $\bar{\partial}^{-1}$ and write the conserved quantity $\mathcal{J}$ in the following form

$$
\mathcal{J}=J+\bar{\partial}^{-1}\left(p^{2} H\right)
$$

where in this notation $\bar{\partial} \bar{\partial}^{-1}=\bar{\partial}^{-1} \bar{\partial}=\mathbf{1}$. 


\section{Integrability Properties.}

It has been shown [4] that the generalized Weierstrass system has the property of integrability when the mean curvature is constant. Now we are interested in conditions under which system (2.1) becomes a completely integrable system. Employing the conditional symmetry method [11], we look for conditions necessary for solvability of a class of equations (3.4) which admit compatible first order differential constraints. We consider here the simplest case where the differential constraints are based on an $\operatorname{sl}(2, \mathbb{C})$ representation. Assume then that they take the form of coupled Riccati equations with nonconstant coefficients and their complex conjugates,

$$
\begin{aligned}
& \partial \rho=A_{1}^{0}(z, \bar{z})+A_{1}^{1}(z, \bar{z}) \rho+A_{1}^{2}(z, \bar{z}) \rho^{2}, \\
& \bar{\partial} \rho=A_{2}^{0}(z, \bar{z})+A_{2}^{1}(z, \bar{z}) \rho+A_{2}^{2}(z, \bar{z}) \rho^{2} .
\end{aligned}
$$

To ensure that the pair of equations in (4.1) satisfies a compatibility condition, it must be required that the coefficient functions satisfy the following system of zero curvature conditions

$$
\begin{gathered}
\bar{\partial} A_{1}^{0}-\partial A_{2}^{0}+A_{1}^{1} A_{2}^{0}-A_{2}^{1} A_{1}^{0}=0, \\
\bar{\partial} A_{1}^{1}-\partial A_{2}^{1}+2 A_{1}^{2} A_{2}^{0}-2 A_{2}^{2} A_{1}^{0}=0, \\
\bar{\partial} A_{1}^{2}-\partial A_{2}^{2}+A_{1}^{2} A_{2}^{1}-A_{1}^{1} A_{2}^{2}=0 .
\end{gathered}
$$

We look for conditions on the function $H$ which ensure that the overdetermined system composed of (3.4), differential constraints (4.1) and conditions (4.2) are in involution. These involutivity conditions give the specific differential restrictions on the function $H$

$$
\bar{\partial} \partial\left(\frac{1}{H}\right)=0 .
$$

A general solution of equation (4.3) is given by

$$
H=\frac{1}{Q(z)+Q(\bar{z})},
$$

where $Q(z)$ is an arbitrary real valued function, and (4.4) is written in this form so that $H$ goes into itself under conjugation. In this case, system (2.1) can be written as

$$
\begin{gathered}
\partial \psi_{1}=\frac{p}{Q(z)+Q(\bar{z})} \psi_{2} \quad \bar{\partial} \bar{\psi}_{1}=\frac{p}{Q(z)+Q(\bar{z})} \bar{\psi}_{2}, \\
\bar{\partial} \psi_{2}=-\frac{p}{Q(z)+Q(\bar{z})} \psi_{1}, \quad \partial \bar{\psi}_{2}=-\frac{p}{Q(z)+Q(\bar{z})} \bar{\psi}_{1} .
\end{gathered}
$$


Another way to write the solution to $(4.3)$ is $H=(h(z)+\bar{h}(\bar{z}))^{-1}$, where $h(z)$ is a holomorphic function. Beginning with the definition of $p$ in (2.1), then modulo system (2.1), it is straightforward to show that

$$
\partial p=\psi_{1} \partial \bar{\psi}_{1}+\bar{\psi}_{2} \partial \psi_{2}, \quad \bar{\partial} p=\bar{\psi}_{1} \bar{\partial} \psi_{1}+\psi_{2} \bar{\partial} \bar{\psi}_{2}
$$

Differentiating $\partial p$ in (4.1) with respect to $\bar{z}$, we obtain an expression for $\bar{\partial} \partial p$ as follows

$$
\begin{gathered}
\bar{\partial} \partial p=\bar{\partial} \psi_{1} \partial \bar{\psi}_{1}+\psi_{1} \bar{\partial} \partial \bar{\psi}_{1}+\bar{\partial} \partial \psi_{2} \bar{\psi}_{2}+\partial \psi_{2} \bar{\partial} \bar{\psi}_{2} \\
=\bar{\partial} \psi_{1} \partial \bar{\psi}_{1}+\psi_{1}\left(\partial p H \bar{\psi}_{2}+p \partial H \bar{\psi}_{2}+p H \partial \bar{\psi}_{2}\right)-\bar{\psi}_{2}\left(\partial p H \psi_{1}+p \partial H \psi_{1}+p H \partial \psi_{1}\right)+\partial \psi_{2} \bar{\partial} \bar{\psi}_{2} \\
=\bar{\partial} \psi_{1} \partial \bar{\psi}_{1}+\partial \psi_{2} \bar{\partial} \bar{\psi}_{2}-p^{3} H^{2}
\end{gathered}
$$

It has been shown that $p$ satisfies a modified sinh-Gordon equation when $H$ is constant [5]. Here, it will be shown that, with respect to system (2.1), $p$ satisfies essentially an identical equation.

Proposition 4. Let $p$ be defined by (2.1) and $J$ defined by (3.7), then $p$ satisfies a second order differential equation which involves $p, J$ and the mean curvature $H$ and is given by

$$
\partial \bar{\partial} \ln p=\frac{|J|^{2}}{p^{2}}-p^{2} H^{2} .
$$

Proof: The derivative $\partial \bar{\partial} \ln p$ is expanded in the form

$$
\partial \bar{\partial} \ln p=\frac{1}{p^{2}}(p \partial \bar{\partial} p-\partial p \bar{\partial} p)
$$

The derivatives on the right hand side have been evaluated and are substituted from (4.1) and (4.2). We obtain that

$$
\begin{gathered}
\partial \bar{\partial} \ln p=\frac{1}{p^{2}}\left(p\left(\bar{\partial} \psi_{1} \partial \bar{\psi}_{1}+\partial \psi_{2} \bar{\partial} \bar{\psi}_{2}\right)-p^{4} H^{2}-\left(\psi_{1} \partial \bar{\psi}_{1}+\bar{\psi}_{2} \partial \psi_{2}\right)\left(\overline{\psi_{1}} \bar{\partial} \psi_{1}+\psi_{2} \bar{\partial} \bar{\psi}_{2}\right)\right) \\
=\frac{1}{p^{2}}\left(\left|\psi_{1}\right|^{2} \partial \psi_{2} \bar{\partial} \bar{\psi}_{2}+\left|\psi_{2}\right|^{2} \bar{\partial} \psi_{2} \partial \bar{\psi}_{1}-\bar{\psi}_{1} \bar{\psi}_{2} \bar{\partial} \psi_{1} \partial \psi_{2}-\psi_{1} \psi_{2} \partial \bar{\psi}_{1} \bar{\partial} \bar{\psi}_{2}-p^{4} H^{2}\right)
\end{gathered}
$$

Using the definition of $J$ given in (3.7), it follows immediately that

$$
|J|^{2}=\left|\psi_{1}\right|^{2} \partial \psi_{2} \bar{\partial} \bar{\psi}_{2}-\psi_{1} \psi_{2} \partial \bar{\psi}_{1} \bar{\partial} \bar{\psi}_{2}-\bar{\psi}_{1} \bar{\psi}_{2} \partial \psi_{2} \bar{\partial} \psi_{1}+\left|\psi_{2}\right|^{2} \partial \overline{\psi_{1}} \bar{\partial} \psi_{1}
$$


Comparing this expression for $|J|^{2}$ with the first four terms in the bracket in (4.9), it follows that $p$ satisfies the equation

$$
p^{2} \partial \bar{\partial} \ln p=|J|^{2}-p^{4} H^{2}
$$

Dividing both sides of this equation by $p^{2}$, we immediately obtain (4.8).

It has been shown [4] that when $H$ is constant, there is a connection between the time independent Landau-Lifshitz equation which takes the form

$$
[S, \partial \bar{\partial} S]=0
$$

and the two-dimensional Euclidean nonlinear sigma model [12-14]. The matrix $S$ will be referred to as the spin matrix. In terms of $\rho$ the sigma model variable, the matrix $S$ takes the form

$$
S=\frac{1}{1+|\rho|^{2}}\left(\begin{array}{cc}
1-|\rho|^{2} & 2 \bar{\rho} \\
2 \rho & -1+|\rho|^{2}
\end{array}\right) .
$$

The sigma model system (3.4) for the case in which $\rho$ is constant can be written in the form

$$
\partial \bar{\partial} \rho-\frac{2 \bar{\rho}}{1+|\rho|^{2}} \partial \rho \bar{\partial} \rho=0, \quad \bar{\partial} \partial \bar{\rho}-\frac{2 \rho}{1+|\rho|^{2}} \bar{\partial} \bar{\rho} \partial \bar{\rho}=0
$$

Define $f$ and $\bar{f}$ to be the $\rho$ dependent factors on the left hand side of each respective equation in (4.12). Then when $H$ is constant, from (4.12) the sigma model equations can be written in the form $f=0, \bar{f}=0$. The matrix generated by (4.10) in terms of $f$ and $\bar{f}$ is given explicitly by

$$
[S, \partial \bar{\partial} S]=\frac{4}{\left(1+|\rho|^{2}\right)^{2}}\left(\begin{array}{cc}
\bar{\rho} f-\rho \bar{f} & \bar{\rho}^{2} f-\bar{f} \\
-\left(f-\rho^{2} \bar{f}\right) & -(\bar{\rho} f-\rho \bar{f})
\end{array}\right) .
$$

We can summarize this in the following Proposition.

Proposition 5. If $\rho$ is a solution of the nonlinear sigma model system (4.12), then the spin matrix $S$ defined by (4.11) is a solution of the Landau-Lifshitz equation (4.10).

Of course, the spin matrix $S$ can be written in terms of $\psi_{1}$ and $\psi_{2}$, and Proposition 5 is altered to require that $\psi_{1}$ and $\psi_{2}$ are solutions of the generalized Weierstrass system, (2.1) with $H$ constant. 
At this point, we would like to adapt this result to the case in which $H$ is not constant. To this end, we consider a nonhomogeneous or deformed analogue of (4.10) given by

$$
[S, \partial \bar{\partial} S]+\mathcal{R H}=0
$$

and the matrices $\mathcal{R}$ and $\mathcal{H}$ are given by

$$
\mathcal{R}=\frac{4}{\left(1+|\rho|^{2}\right)^{2}}\left(\begin{array}{cc}
-\bar{\rho} \partial \rho & \rho \bar{\partial} \bar{\rho} \\
\partial \rho & -\rho^{2} \bar{\partial} \bar{\rho}
\end{array}\right), \quad \mathcal{H}=\left(\begin{array}{cc}
\bar{\partial} \ln H & \bar{\rho} \bar{\partial} \ln H \\
\partial \ln H & \frac{1}{\rho} \partial \ln H
\end{array}\right)
$$

where the matrix $\mathcal{R}$ depends only on the $\rho$ variable. It is straightforward to calculate the product $\mathcal{R H}$ and so (4.13) takes the form

$$
\begin{aligned}
& {[S, \partial \bar{\partial} S]+\mathcal{R} \mathcal{H}=\frac{4}{\left(1+|\rho|^{2}\right)^{2}}} \\
& \cdot\left(\begin{array}{cc}
\bar{\rho}(f-(\bar{\partial} \ln H) \partial \rho)-\rho(\bar{f}-(\partial \ln H) \bar{\partial} \bar{\rho}) & \left.\bar{\rho}^{2}(f-(\bar{\partial} \ln H) \partial \rho)-(\bar{f}-(\partial \ln H) \bar{\partial} \bar{\rho})\right) \\
-\left(f-(\bar{\partial} \ln H) \partial \rho-\rho^{2}(\bar{f}-(\partial \ln H) \bar{\partial} \bar{\rho})\right) & -(\bar{\rho}(f-(\bar{\partial} \ln H) \bar{\partial} \rho)-\rho(\bar{f}-(\partial \ln H) \bar{\partial} \bar{\rho}))
\end{array}\right)
\end{aligned}
$$

This gives the following generalization of Proposition 5.

Proposition 6. If $\rho$ is a solution of nonlinear sigma model equations (3.4), and the matrices $\mathcal{R}$ and $\mathcal{H}$ in (4.13) are defined by (4.14), then the spin matrix $S$ given in (4.11) is a solution of the nonhomogeneous Landau-Lifshitz equation (4.13) modulo (3.4).

In analogy with the constant mean curvature case, there is a set of conditions which permit the system (2.1) to become a linear decoupled system of equations which have nonconstant coefficients.

Proposition 7: If the functions $\psi_{1}$ and $\psi_{2}$ satisfy the overdetermined system composed of the equations of motion (2.1) and differential constraints

$$
\bar{\psi}_{1} \bar{\partial} \psi_{1}+\psi_{2} \bar{\partial} \bar{\psi}_{2}=0, \quad \bar{\psi}_{2} \partial \psi_{2}+\psi_{1} \partial \bar{\psi}_{1}=0
$$

then the overdetermined system is equivalent to a linear system with nonconstant coefficients of the form

$$
\bar{\partial} \partial \psi_{1}-(\bar{\partial} \ln H) \partial \psi_{1}+p_{0}^{2} H^{2} \psi_{1}=0, \quad \partial \bar{\partial} \psi_{2}-(\partial \ln H) \bar{\partial} \psi_{2}+p_{0}^{2} H^{2} \psi_{2}=0
$$


and their respective conjugate equations, where $\left|\psi_{1}\right|^{2}+\left|\psi_{2}\right|^{2}=p_{0}^{2} \in \mathbb{R}$.

Proof: Making use of (4.6) and the conditions (4.16), we obtain that the derivatives of $p$ vanish, $\partial p=0$ and $\bar{\partial} p=0$. This means that if $(4.16)$ hold, then $p$ is a real constant $p_{0}$. Thus, $\left|\psi_{1}\right|^{2}+\left|\psi_{2}\right|^{2}=p_{0}$ is a conserved quantity. Differentiating system (2.1) and replacing the known derivatives, we obtain the second order system (4.17).

\section{Determination of Specific Solutions.}

In this section, we would like to determine specific examples of solutions for system (2.1). To this end, system (3.4) can be exploited to determine functions $\rho$ corresponding to the given function $H(z, \bar{z})$, which describes the mean curvature of the surface. Once these functions $\rho$ have been obtained, equations (3.3) are used to determine $\psi_{1}$ and $\psi_{2}$ from $\rho$, and (2.2) then produces the coordinates of the associated surface of mean curvature $H$.

A specific class of solution to (3.4) has been extensively investigated $[5,6]$ in the case in which the mean curvature is constant. This concerns the case in which $\rho$ satisfies $|\rho|^{2}=1$, that is, solutions which are unimodular. Such solutions lead in a straightforward way to the construction of multisoliton solutions. It is worth showing here that such solutions exist only for the case in which the mean curvature $H$ is constant.

Proposition 8. Suppose $\rho$ is a solution of the sigma model system (3.4) which satisfies the condition $|\rho|^{2}=1$. Then it follows that the mean curvature function $H$ in (3.4) is a constant.

Proof: Substituting $|\rho|^{2}=1$ into system (3.4) it reduces to

$$
\bar{\partial} \ln \rho-\bar{\rho} \bar{\partial} \rho=\bar{\partial} \ln H, \quad \partial \ln \bar{\rho}-\rho \partial \bar{\rho}=\partial \ln H
$$

Integrating each of the equations in (5.1) in turn with respect to $\bar{z}$ and $z$ respectively, we obtain

$$
\ln \rho-\int \bar{\rho} \bar{\partial} \rho d \bar{z}+f(z)=\ln H, \quad \ln \bar{\rho}-\int \rho \partial \bar{\rho} d z+g(z)=\ln H .
$$

Therefore $H$ must be given equivalently in the form

$$
H=\rho e^{-\int \bar{\rho} \bar{\partial} \rho d \bar{z}+f(z)}, \quad H=\bar{\rho} e^{-\int \rho \partial \bar{\rho} d z+g(z)}
$$

Now substitute $\bar{\rho}=1 / \rho$ into (5.3) and simplify to obtain

$$
H=\rho e^{-\int \bar{\partial} \ln \rho d \bar{z}+f(z)}=\rho e^{-\ln \rho+f(z)}=e^{f(z)}, \quad H=\bar{\rho} e^{\int \partial \ln \rho d z+g(\bar{z})}=\bar{\rho} e^{\ln \rho+g(\bar{z})}=e^{g(\bar{z})}
$$


Since these have to give the same real valued function $H, f$ and $g$ must be equal to the same real constant $f(z)=g(\bar{z})=c$, which implies that $H$ is constant.

When the mean curvature $H$ is constant, the following Proposition gives a way of constructing multisoliton solutions from individual unimodular solutions [5].

Proposition 9. If $\rho_{1}$ and $\rho_{2}$ are unimodular and as well satisfy the sigma model system (4.12) for constant $H$, then the product of the two functions $\rho=\rho_{1} \cdot \rho_{2}$ also satisfies the sigma model system.

Proposition 10. Let $H$ be a mean curvature function which is identically constant and $\varphi$ a function which satisfies the pair of relations

$$
\partial \varphi=\ln (\partial \ln \rho), \quad \bar{\partial} \varphi=\ln H
$$

where $\rho$ is unimodular. The compatibility condition for (5.4) is equivalent to the sigma model system (4.12).

Proof. Differentiating the first equation in (5.4) with respect to $\bar{z}$ and the second with respect to $z$, we obtain the results

$$
\bar{\partial} \partial \varphi=\frac{\bar{\partial} \partial \rho}{\partial \rho}-\frac{\bar{\partial} \rho}{\rho}, \quad \partial \bar{\partial} \varphi=0 .
$$

Equating these, multiplying both sides by $\partial \rho$ and applying the constraint $\bar{\rho}=1 / \rho$, it is found that $\rho$ satisfies the first equation in (4.12). Using the complex conjugates of (5.4), the second equation in (4.12) is obtained in the same way.

Specific solutions to system (3.4) are more difficult to determine than in the case of constant mean curvature, however, in what follows a few examples are given.

(i) As a simple example to begin with, consider mean curvature $H$ given by the rational function

$$
H(z, \bar{z})=\frac{1}{1+\lambda^{2}(z+\bar{z})^{2}}
$$

$\lambda$ is a real constant, and $H$ is real-valued and analytic. By substituting $H$ into (3.4), it can be checked that the function

$$
\rho=\lambda(z+\bar{z})=\bar{\rho},
$$


is a solution to system (3.4). Moreover, $\partial \rho=\lambda=\bar{\partial} \bar{\rho}$ and the corresponding solution to system (2.1) is given by

$$
\psi_{1}=\epsilon \frac{\lambda^{3 / 2}(z+\bar{z})}{\left(1+\lambda^{2}(z+\bar{z})^{2}\right)^{1 / 2}}, \quad \psi_{2}=\epsilon \frac{\lambda^{1 / 2}}{\left(1+\lambda^{2}(z+\bar{z})^{2}\right)^{1 / 2}}, \quad \epsilon= \pm 1 .
$$

Then (2.2) induces a surface with mean curvature function given in (5.5).

(i) Let the mean curvature function be given as follows

$$
H(z, \bar{z})=\frac{\exp (\lambda(z+\bar{z}))}{1+\exp (\lambda(z+\bar{z}))^{2}}
$$

where $\lambda$ is an arbitrary real nonzero constant. In this case, $H$ is real valued and analytic. Substituting (5.8) into (3.4), it can be verified that the function $\rho$ given by

$$
\rho=\exp (\lambda(z+\bar{z}))=\bar{\rho}
$$

is a solution to system (3.4). From (5.9), we calculate

$$
\partial \rho=\lambda \exp (\lambda(z+\bar{z}))=\bar{\partial} \bar{\rho}
$$

and from (3.3), the functions $\psi_{i}$ are given by

$$
\psi_{1}=\epsilon \exp (\lambda(z+\bar{z})) \frac{\lambda^{1 / 2}}{(1+\exp (2 \lambda(z+\bar{z})))^{1 / 2}}, \quad \psi_{2}=\epsilon \frac{\lambda^{1 / 2}}{(1+\exp (2 \lambda(z+\bar{z})))^{1 / 2}}, \quad \epsilon= \pm 1 .
$$

The $\psi_{i}$ in (5.11) can be used to determine the coordinates of a surface by means of the inducing prescription (2.2) corresponding to the function $H$ in (5.8).

(iii) As a final example, let the mean curvature function be given by

$$
H=A \tan (A(z+\bar{z})) \frac{\cos ^{2}(A(z+\bar{z}))+2}{\cos ^{2}(A(z+\bar{z}))-2}
$$

where $A$ is an arbitrary, real nonzero constant, so that $H$ is real valued. With (5.12) placed in $(3.4)$, it is found that

$$
\rho=\sin (A(z+\bar{z}))=\bar{\rho}
$$

is a solution to system (3.4). From (5.13), we have that

$$
\partial \rho=A \cos (A(z+\bar{z}))=\bar{\partial} \bar{\rho} .
$$


The corresponding solutions to (2.1) are then

$$
\psi_{1}=\epsilon \sin (A(z+\bar{z})) \frac{(A \cos (A(z+\bar{z})))^{1 / 2}}{H^{1 / 2}\left(1+\sin ^{2}(A(z+\bar{z}))\right)}, \quad \psi_{2}=\epsilon \frac{(A \cos (A(z+\bar{z})))^{1 / 2}}{H^{1 / 2}\left(1+\sin ^{2}(A(z+\bar{z}))\right)}, \quad \epsilon= \pm 1 .
$$

The coordinates of the surface follow by using (2.2) with the $\psi_{i}$ given in these three examples (i)-(iii).

\section{Summary and Physical Application.}

In this paper, an investigation of the generalized Weierstrass system for the case in which the mean curvature of the surface is not constant has been initiated. It has been shown, for example, that there exists a transformation from solutions of the generalized Weierstrass system (2.1) to solutions of a nonlinear sigma model system (3.4), and of course, a transformation from solutions of (3.4) to solutions of system (2.1). We would like to conclude by presenting a few remarks as to how the ideas described here can be brought to bear on the study of string theory.

A rigid string action can be written in the form $[15,16]$

$$
A=\gamma \iint d S+\alpha \iint H^{2} d S
$$

where $\alpha$ and $\gamma$ are some constants, and integration takes place over the string world surface $S$ which has extrinsic mean curvature $H$. Moreover, one can consider restricting ourselves to threedimensional space-time and to a Euclidean version of the model. By varying the function $A$, very simple equations relating fundamental geometrical invariants of the string world surface can be obtained. The Euler-Lagrange equation following from the vanishing of the normal variation of the rigid string action is found to be given by

$$
-2 \gamma H+\alpha\left(\Delta H+2 H^{3}+R H\right)=0
$$

where $H$ is the mean curvature, $R$ is the scalar curvature which is related to the Gauss curvature by $K=-R / 2$ and $\Delta$ is the Laplace-Beltrami operator given on the surface $M$.

The theory described here then provides a connection between the solution of an equation of motion and a method whereby the coordinate expressions of an actual surface can be calculated 
using (2.2), in a straightforward way. It is hoped that further work will yield further results in higher dimensional spaces, such as Minkowski space relatively soon.

\section{References.}

[1] K. Weierstrass, Fortsetzung der Untersuchung über die Minimalflächen, Mathematische Werke, Vol. 3 (Verlagsbuchhandlung, Hillesheim, 1866) pp. 219-248; G. Darboux, Lecons sur Systèmes Orthogonaux et les Coordonnes Curvilignes (Gauthier-Villars, Paris, 1910).

[2] B. Konopelchenko, "Induced surfaces and their integrable dynamics," Stud. Appl. Math. 96, 9-51 (1996).

[3] B. G. Konopelchenko and I. A. Taimanov, "Constant mean curvature surfaces via an integrable dynamical system," J. Phys. A 29, 1261-1265 (1996).

[4] P. Bracken, A. M. Grundland and L. Martina, "The Weierstrass-Enneper System for Constant Mean Curvature Surfaces and the Completely Integrable Sigma Model," J. Math. Phys. 40, 3379-3402 (19999).

[5] P. Bracken and A. M. Grundland, "Symmetry Properties and Explicit Solutions of the Generalized Weierstrass System", J. Math. Phys. 42, 1250-1282 (2001).

[6] P. Bracken and A. M. Grundland, "On Certain Classes of Solutions of the Weierstrass-Enneper System Inducing Constant Mean Curvature Surfaces", J. of Nonlinear Mathematical Physics, 6, 294-313 (1999).

[7] P. Bracken and A. M. Grundland, "On Complete Integrability of the Generalized Weierstrass System", J. of Nonlinear Mathematical Physics, 9, 229-247 (2002).

[8] W. Zakrzewski, Low Dimensional Sigma-Models (Hilger, New York, 1989).

[9] D. Nelson, T. Piran, and S. Weinberg, Statistical Mechanics of Membranes and Surfaces (World Scientific, Singapore, 1992).

[10] D. G. Gross, C. N. Pope, and S. Weinberg, Two-dimensional Quantum Gravity and Random Surfaces (World Scientific, Singapore, 1992).

[11] A. M. Grundland, L. Martina and G. Rideau, Partial differential equations with differential constraints, in CRM Proceedings and Lecture Notes (American Mathematical Society, Providence, 
RI, 1997), Vol. 11, pp. 135-154.

[12] V. G. Makhankov and O. K. Pashaev, Integrable Pseudospin Models in Condensed Matter, Sov. Sci. Rev. Math. Phys. 9, 1-151 (1992).

[13] P. Bracken, "Spin Model Equations, Connections with Integrable Systems and Applications to Magnetic Vortices", Int. J. of Mod. Physics, B 17, 4325-4537 (2003).

[14] P. Bracken, "Reductions of Chern-Simons Theory to Integrable Systems which have Geometric Applications", Int. J. Mod. Physics B 18, 1261-1275 (2004).

[15] A. Polyakov, "Fine structure of strings", Nucl. Phys. B 286, 406-412, (1986).

[16] H. Kleinert, "The membrane properties of condensing strings", Phys. Letts B 174, 335-338, (1986). 